\title{
DOTATIONS EN CAPITAL ET PAUVRETÉ DES MÉNAGES AU BURKINA FASO : UNE ANALYSE EN ÉQUILIBRE GÉNÉRAL CALCULABLE
}

\author{
Jean-Marc Montaud
}

De Boeck Université | Revue d'économie du développement

\author{
2003/1 - Vol. 17 \\ pages 43 à 71
}

ISSN 1245-4060

ISBN 2-8041-4208-6

Article disponible en ligne à l'adresse:

http://www.cairn.info/revue-d-economie-du-developpement-2003-1-page-43.htm

Pour citer cet article :

Montaud Jean-Marc, « Dotations en capital et pauvreté des ménages au Burkina Faso : une analyse en Équilibre Général Calculable »,

Revue d'économie du développement, 2003/1 Vol. 17, p. 43-71. DOI : 10.3917/edd.171.0043

Distribution électronique Cairn.info pour De Boeck Université.

(c) De Boeck Université. Tous droits réservés pour tous pays.

La reproduction ou représentation de cet article, notamment par photocopie, n'est autorisée que dans les limites des conditions générales d'utilisation du site ou, le cas échéant, des conditions générales de la licence souscrite par votre établissement. Toute autre reproduction ou représentation, en tout ou partie, sous quelque forme et de quelque manière que ce soit, est interdite sauf accord préalable et écrit de l'éditeur, en dehors des cas prévus par la législation en vigueur en France. II est précisé que son stockage dans une base de données est également interdit. 


\title{
Dotations en capital et pauvreté des ménages au Burkina Faso : une analyse en Équilibre Général Calculable
}

Capital Endowment and Household Poverty in

Burkina Faso : A Computable General Equilibrium Model

\author{
Jean-Marc Montaud \\ Maître de Conférences au CATT de \\ l'Université de Pau et des Pays de l'Adour
}

Membre associé du CED de l'Université Montesquieu-Bordeaux IV

\begin{abstract}
Cette analyse tente d'explorer les déterminants de la pauvreté au Burkina Faso à l'aide d'un modèle d'Équilibre Général Calculable (EGC) et d'une désagrégation des ménages reposant sur leurs dotations respectives en capital économique ou social. Les données utilisées sont celles de l'enquête nationale sur les ménages effectuée dans le pays en 1994-1995. À partir de l'identification de différents groupes de ménages et de la mesure de leurs niveaux de pauvreté et de vulnérabilité initiaux, nous élaborons une Matrice de Comptabilité Sociale du Burkina Faso. Sur cette base empirique, nous simulons, grâce à un modèle EGC, plusieurs chocs exogènes ou politiques économiques et nous montrons leurs impacts sur la pauvreté des différents groupes de ménages dans ce pays.
\end{abstract}

Classification JEL : C68, D58, I32, O15

Mots clés : Équilibre Général Calculable, Pauvreté, Burkina Faso

Our purpose is to examine the factors of poverty in Burkina Faso within a Computable General Equilibrium (CGE) framework. The households are desagregated upon their respective endowment of economical and social capital. The data used are those of the household survey done in Burkina Faso in 1994-1995. These data allows identifying different household and defining their poverty and vulnerability profile. They are integrated in a Social Acounting Matrix of Burkina Faso witch is the database for a CGE model destinated to show the impact of macro-shocks or policies on household's poverty in this country. 


\section{INTRODUCTION}

Depuis le début des années quatre-vingt, le Burkina Faso présente des taux de croissance réels relativement élevés ${ }^{1}$, récoltant ainsi les fruits d'une gestion prudente en matière de finances publiques et de la mise en place de programmes de stabilisation et de réformes structurelles. Toutefois, malgré ces résultats macroéconomiques encourageants, le bilan social de la décennie quatre-vingt-dix s'avère largement négatif dans ce petit pays sahélien qui, entre 1993 et 2000, est passé du $170^{\text {ème }}$ au $172^{e ̀ m e}$ rang mondial (sur 174) en termes de développement humain ${ }^{2}$. Toutes les analyses empiriques confirment en effet que les conditions de vie de la population n'ont cessé de se dégrader au cours de la décennie ${ }^{3}$. Le Cadre Stratégique de Lutte contre la Pauvreté (CSLP) du Burkina $\mathrm{Faso}^{4}$ souligne par exemple que les principaux indicateurs d'éducation, de santé et d'accès aux services publics se détériorent ou restent à des niveaux très insuffisants. Entre 1994 et 1998, les taux d'analphabétisme sont passés de $81,1 \%$ à $81,6 \%$. L'espérance de vie moyenne de la population atteint à peine 54 ans en 1999 et les taux de mortalité des enfants de moins de cinq ans sont passés de 204,5\%o à 219\%o entre 1993 et 1999. L'approvisionnement de la population en eau potable progresse mais reste insuffisant malgré les efforts d'équipement du pays et, en 1994 comme en 1998, moins de 1\% des ménages ruraux disposaient de l'électricité dans leur logement.

Dans ce contexte défavorable, le CSLP souligne l'ampleur et l'aggravation de la pauvreté des ménages durant la dernière décennie au Burkina Faso. Malgré la croissance économique, les taux d'incidence de la pauvreté sont passés de 44,5\% à 45,3\% entre 1994 et $1998^{5}$. Cette progression n'a toutefois pas été homogène parmi la population, certains types d'individus connaissant une forte dégradation de leurs situations relatives. C'est le cas de ceux vivant dans les zones urbaines ${ }^{6}$ ou dans les régions les moins fertiles et les moins bien dotées en ressources naturelles du centre du pays, ou encore de ceux appartenant à certains

1 De près de $3 \%$ entre 1980 et 1993 , les taux de croissance annuels moyens sont passés à près de $5 \%$ entre 1994 et 2000 .

2 PNUD (1993, 2000).

3 Voir par exemple Lachaud (1997, 1999, 2000, 2001), Raffinot (1997), Montaud (2002).

4 Ministère de l'Économie, des Finances et du Plan du Burkina Faso (2000).

5 Estimations obtenues à partir des données des deux enquêtes prioritaires de 1994 et 1998 et sur la base d'une ligne de pauvreté de 41099FCFA en 1994 et 72690FCFA en 1998. Nos propres calculs effectués à partir des ménages et non des individus montrent des taux d'incidence de la pauvreté élevés mais stables de 32,5\% en 1994 et 32,4\% en 1998. Voir Montaud (2002).

6 Malgré cette progression, la contribution de la pauvreté urbaine à la pauvreté totale reste encore faible (3,8\% en 1994 et $6,1 \%$ en 1998). 
groupes socio-économiques particuliers, comme, par exemple, les agriculteurs vivriers.

Face à ces constats, il nous a semblé opportun d'essayer de mieux comprendre les facteurs explicatifs de l'ampleur et des mécanismes de diffusion de la pauvreté au sein de la population burkinabé. Pour cela, nous avons choisi d'orienter l'analyse autour de deux axes principaux. En premier lieu, nous nous inscrivons dans la lignée des travaux théoriques qui utilisent les modèles d'Équilibre Général Calculable (EGC) pour l'analyse de la pauvreté ${ }^{7}$. L'objectif est ici de replacer le phénomène dans le fonctionnement global de l'économie burkinabé et de prendre en compte l'ensemble des interdépendances entre l'offre, les revenus et la demande pour évaluer les conséquences de différents chocs macroéconomiques sur le niveau de pauvreté des ménages. En second lieu, face au renouveau de la réflexion conceptuelle autour de la pauvreté ${ }^{8}$, nous avons pris le parti d'élargir le cadre traditionnel de l'analyse de la pauvreté monétaire en faisant reposer la désagrégation des ménages sur le critère multidimensionnel de la vulnérabilité. Celle-ci peut se définir à partir des dotations du ménage en actifs de différentes natures, économiques mais également sociologiques, et indique sa capacité à plus ou moins bien résister au risque de tomber dans la pauvreté à la suite d'un choc exogène défavorable ${ }^{9}$.

La première section est destinée à définir ces choix méthodologiques et à en préciser leurs fondements. La deuxième section part de données d'enquêtes pour désagréger les ménages du Burkina Faso en différentes catégories selon le critère de leur vulnérabilité et caractériser leur situation initiale en termes de pauvreté et d'inégalités. La troisième section décrit les principales caractéristiques du modèle EGC utilisé et la section suivante précise les modalités d'élaboration de la Matrice de Comptabilité Sociale qui lui sert de support. Une fois ces étapes franchies, il est enfin possible, dans la dernière section, de simuler l'impact de plusieurs chocs macroéconomiques sur la pauvreté des différents groupes de ménages au Burkina Faso.

7 Voir, par exemple, Decaluwé, Dumont et Savard (1999), Decaluwé, Patry et Savard (1998), Decaluwé, Patry, Savard et Thorbecke (1999) ou encore Robilliard (2000).

8 Sen (1985,1992), Banque Mondiale (1990, 2001), Ravallion (1998).

9 Moser (1996). 


\section{CONCEPTS ET MÉTHODES}

Si l'utilisation de modèles EGC pour l'analyse de la distribution intergroupes des revenus dans les PED n'est pas nouvelle ${ }^{10}$, leur application à l'analyse de la pauvreté est en revanche plus récente. L'intérêt d'un tel outil est de relier directement le phénomène aux modes de formation des revenus et des prix relatifs dans l'économie. Il est en effet possible de déterminer l'influence d'un choc exogène ou d'une politique macroéconomique sur la pauvreté de différents groupes de ménages grâce à l'analyse des variations simultanées de leurs niveaux de consommation respectifs, qui dépendent de l'évolution des revenus, et de la ligne de pauvreté, qui dépend de l'évolution des prix. La difficulté principale d'un tel exercice réside toutefois dans l'obligation de s'affranchir de l'hypothèse néo-classique de l'agent représentatif. Cette hypothèse n'autorise en effet qu'une analyse inter-groupes des distributions de revenus, alors que l'utilisation des indicateurs traditionnels de la pauvreté repose sur une analyse intra-groupe des inégalités ${ }^{11}$. À ce stade, deux voies de recherche ont été explorées dans la littérature économique. La première, dite de «micro-simulation », consiste à minimiser le problème en désagrégeant le plus possible la population des ménages à partir des données d'enquêtes ${ }^{12}$. Cette approche combine donc la modélisation EGC traditionnelle avec une analyse économétrique des comportements microéconomiques des ménages enquêtés, qui deviennent ainsi autant d'agents représentatifs dans le modèle. Si elle permet d'obtenir des résultats satisfaisants ${ }^{13}$, elle reste toutefois lourde en termes de collecte et de traitement des données. Pour contourner cette difficulté, une seconde voie de recherche préfère supposer la constance de la distribution intra-groupe entre deux équilibres. Autrement dit, les différents groupes de ménages sont supposés suffisamment homogènes pour qu'un choc exogène affecte la distribution inter-groupes des revenus mais pas les distributions intragroupe $^{14}$. C'est cette hypothèse que nous retiendrons ici. De l'avis même des auteurs, ce postulat ad hoc constitue une limite méthodologique certaine. Selon nous, elle peut toutefois être minimisée en accordant une attention toute particulière aux critères de désagrégation retenus pour les ménages. À ce stade, seule une désagrégation reposant sur la pro-

10 Voir, par exemple, Adelman et Robinson (1979), Dervis et al (1982), ou encore Morrisson (1992).

11 Indicateurs de la classe F-G-T, Foster, Greer et Thorbecke (1984).

12 Cogneau (1997), Cogneau et Robilliard (1999), Robilliard (2000).

13 Pour une comparaison des différentes méthodologies, voir Decaluwé, Dumont et Savard (1999) ou Robilliard (2000).

14 Decaluwé, Dumont et Savard (1999), Decaluwé, Patry et Savard (1998), Decaluwé, Patry, Savard et Thorbecke (1999). 
blématique de la pauvreté apparaît alors ici véritablement pertinente. Il s'agit en effet de garantir des degrés de différenciation inter-groupes et des degrés d'homogénéité intra-groupe suffisants pour s'assurer a priori que les ménages d'un même groupe seront affectés de manière similaire par un choc exogène en termes de pauvreté. C'est dans cette optique que nous avons choisi de privilégier le concept de vulnérabilité.

Envisagée dans son sens le plus large, la vulnérabilité peut s'interpréter comme le degré de difficulté qu'un individu éprouve pour maintenir le niveau de son bien-être dans un contexte défavorable ${ }^{15}$. Dans le cadre de cette analyse, elle sera comprise comme la capacité de résistance des différents groupes de ménages face au risque de pauvreté. Moser (1996) souligne que ce degré de vulnérabilité dépend étroitement des dotations respectives des ménages en actifs de différente nature tels que le capital physique, le capital humain ou encore le capital social. Cette approche offre l'avantage d'être compatible avec les fondements walrassiens des modèles EGC car elle recentre l'attention sur l'influence des dotations initiales en facteurs dans l'allocation inter-groupes des ressources. Mais cette approche permet surtout de rejoindre les efforts théoriques de ces dernières années pour élargir le champ des interrogations concernant la pauvreté. Il ne s'agit plus de la considérer comme un état statique et unifactoriel mais comme un processus dynamique et multidimensionnel dépassant le simple problème de la répartition des revenus entre les agents. Dans un groupe donné, il est finalement aussi intéressant d'évaluer le niveau de pauvreté des ménages à un instant donné que leur risque de tomber dans une situation de pauvreté. Le degré de ce risque dépend alors de la vulnérabilité de chaque ménage et, à travers elle, de leurs dotations respectives en capital. À cet égard, parmi les différents actifs déterminants de la vulnérabilité, la notion de capital social enrichit l'analyse économique traditionnelle d'une dimension sociologique appréciable. Pouvant être envisagé comme le système de relations dont bénéficie chaque individu, le capital social est en effet certainement déterminant dans la capacité de chacun à résister à la pauvreté, notamment dans un contexte africain où les mécanismes de réciprocités et de coopérations sont extrêmement présents dans la société ${ }^{16}$. L'efficacité de ce capital social apparaît donc ici largement influencée par la prégnance d'une forte culture identitaire communautaire ou ethnique. Elle est également dépendante de la nature des mutations socio-économiques qui accompagnent le processus de développement. L'un des défis de cette

15 Moser (1996), Lachaud (1999).

16 Dans une approche voisine, Mahieu (1990) parle d'un véritable système de droits et d'obligations dans les sociétés africaines. 
analyse sera d'avoir une approche monétaire de cette notion de capital social au sein d'un modèle EGC.

\section{CARACTÉRISATION DES DIFFÉRENTS GROUPES DE MÉNAGES AU BURKINA FASO ET DE LEUR SITUATION INITIALE FACE À LA PAUVRETÉ}

Dans un premier temps, l'identification de différents sous-groupes de ménages a été faite à partir des données de l'enquête nationale prioritaire burkinabé de 1994-1995 $5^{17}$. Cette enquête couvre environ 30000 individus et fournit, entre autres renseignements, des informations sur l'emploi, les revenus ou encore les dépenses de consommation de plus de 8700 ménages. La nomenclature retenue s'inspire de celle de Lachaud (1997). L'emploi étant considéré comme l'actif le plus déterminant de la vulnérabilité des individus, c'est le mode de participation du chef de ménage sur le marché du travail qui en constitue le critère principal. $\mathrm{Au}$ final, huit groupes de ménages distincts sont retenus. Les salariés sont scindés entre «Salariés protégés (1)》 et «Salariés non protégés (2) » selon le caractère permanent ou non de leur emploi, leur cotisation éventuelle à un organisme de retraite, leur situation administrative vis à vis du régime de protection sociale ou leurs droits effectifs aux congés payés. Les indépendants non agricoles sont subdivisés en fonction de la taille de l'entreprise où ils exercent, supposée révélatrice de leurs perspectives de croissance et donc des potentialités d'évolution des indépendants concernés. Ce seuil de taille est fixé arbitrairement à cinq salariés et permet de distinguer les "Indépendants non agricoles évolutifs (3)» des «Indépendants non agricoles involutifs (4)». Les actifs agricoles sont les actifs les plus nombreux ( $72 \%$ de la population totale). Trois sous-groupes homogènes sont identifiés en leur sein à partir d'une procédure d'analyse de données en classification de groupe: les «Agriculteurs progressifs (5)», les «Agriculteurs de subsistance (6) » et les «Éleveurs (7) ». Enfin, une dernière catégorie, «Autres (8)», rassemble les autres groupes de ménages distingués par Lachaud $(1997)^{18}$. Les principales caractéristiques socio-économiques de ces différents groupes de ménages sont exposées dans le tableau 1.

17 INSD (1996).

18 Il s'agit des chômeurs, des autres actifs, c'est-à-dire essentiellement les aides familiaux, et des inactifs. Il n'apparaissait pas en effet véritablement pertinent de les désagréger dans le cadre du modèle EGC du fait de leur situation particulière par rapport au système productif. 
Tableau 1 : Caractéristiques socio-économiques des différents groupes de ménages au Burkina Faso

\begin{tabular}{|c|c|c|c|c|}
\hline Type de ménage & $\begin{array}{c}\text { Salariés } \\
\text { protégés } \\
\text { (1) }\end{array}$ & $\begin{array}{l}\text { Salariés } \\
\text { non } \\
\text { protégés } \\
(2)\end{array}$ & $\begin{array}{c}\text { Indépendants } \\
\text { non agricoles } \\
\text { évolutifs } \\
(3)\end{array}$ & $\begin{array}{c}\text { Indépendants } \\
\text { non agricoles } \\
\text { involutifs } \\
\text { (4) }\end{array}$ \\
\hline \multicolumn{5}{|l|}{ Caractéristiques du ménage } \\
\hline Part dans la population totale ${ }^{1}$ & 6.5 & 3.8 & 0.6 & 7.3 \\
\hline Part dans le revenu total des ménages ${ }^{1}$ & 26,7 & 7,7 & 4,6 & 11,2 \\
\hline Classement selon le revenu moyen ${ }^{2}$ & 2 & 3 & 1 & 4 \\
\hline \multicolumn{5}{|l|}{ Origine ethnique $^{1}$} \\
\hline Mossi & 56.3 & 69.3 & 66.0 & 58.4 \\
\hline Dioula & 39.1 & 24.8 & 22.6 & 33.5 \\
\hline Peuhl & 3.2 & 5.5 & 9.6 & 7.8 \\
\hline \multicolumn{5}{|l|}{ Région d'appartenance $^{1}$} \\
\hline Zone urbaine & 67.6 & 76.3 & 66.0 & 63.9 \\
\hline Zone rurale & 32.4 & 23.7 & 34.0 & 36.1 \\
\hline \multicolumn{5}{|l|}{ Type de Ménage ${ }^{1}$} \\
\hline Monoparental & 12.2 & 16.3 & 6.4 & 20.6 \\
\hline Nucléaire & 30.0 & 40.3 & 25.5 & 35.1 \\
\hline Élargi & 57.8 & 43.3 & 68.1 & 44.3 \\
\hline Taille moyenne ${ }^{3}$ & 5.8 & 5.0 & 9.5 & 5.4 \\
\hline \multicolumn{5}{|l|}{ Caractéristiques du chef de ménage } \\
\hline Âge moyen ${ }^{4}$ & 36 & 35 & 41 & 40 \\
\hline \multicolumn{5}{|l|}{ État civil $^{1}$} \\
\hline Homme & 93.4 & 91.6 & 97.9 & 77.5 \\
\hline Femme & 6.6 & 8.4 & 2.1 & 22.5 \\
\hline Marié & 79.5 & 74.7 & 85.1 & 71.1 \\
\hline \multicolumn{5}{|l|}{ Niveau d'éducation ${ }^{1}$} \\
\hline Aucun & 16.1 & 46.5 & 61.7 & 66.9 \\
\hline Primaire & 18.8 & 26.4 & 23.4 & 23.0 \\
\hline Secondaire premier cycle & 7.2 & 4.3 & 0.0 & 3.3 \\
\hline Secondaire second cycle & 36.0 & 15.7 & 4.3 & 4.7 \\
\hline Supérieur & 12.8 & 4.3 & 6.4 & 1.2 \\
\hline Enseignement Professionnel $(<B E P)$ & 3.4 & 2 & 2.1 & 0.6 \\
\hline Enseignement Professionnel (>BEP) & 5.9 & 0.7 & 2.1 & 0.3 \\
\hline Ancienneté emploi ${ }^{4}$ & 11.3 & 11.0 & 10.7 & 9.8 \\
\hline Exercice d'un emploi secondaire ${ }^{1}$ & 12.6 & 19.1 & 1.3 & 25.8 \\
\hline Propriété des terres cultivables ${ }^{1}$ & 13.6 & 22.7 & 23.4 & 28.3 \\
\hline \multicolumn{5}{|c|}{${ }^{1}$ Pourcentages, ${ }^{2}$ Rang, ${ }^{3}$ Nombre d'individus, ${ }^{4}$ Années } \\
\hline
\end{tabular}


Tableau 1 : Caractéristiques socio-économiques des différents groupes de ménages au Burkina Faso (suite)

Type de ménage

Agriculteurs Agriculteurs Éleveurs Autres Ensemble progressifs de subsistance

(5)

(6)

(7)

(8)

\section{Caractéristiques du ménage}

Part dans la population totale ${ }^{1}$

Part dans le revenu total des ménages ${ }^{1}$

\section{3}

10,3

Classement selon le revenu moyen ${ }^{2}$

Origine ethnique ${ }^{1}$

Mossi
Dioula
Peuhl

Région d'appartenance ${ }^{1}$

Zone urbaine
Zone rurale

Type de Ménage ${ }^{1}$

Monoparental
Nucléaire
Elargi

Taille moyenne ${ }^{3}$

\section{Caractéristiques du chef de ménage}

Âge moyen ${ }^{4}$

État civil ${ }^{1}$

Homme
Femme
Marié

Niveau d'éducation ${ }^{1}$

Aucun

Secondaire premier cycle

Secondaire second cycle

Supérieur

Enseignement Professionnel $(<B E P)$

Enseignement Professionnel ( $>B E P$ )

Ancienneté emploi ${ }^{4}$

Exercice d'un emploi secondaire ${ }^{1}$

Propriété des terres cultivables ${ }^{1}$
6

$$
54.0
$$

43.7

1.9

1.4

98.6

1.5

40.5

58.0

9.7

46

99.0

1.0

93.7

88.2

10.7

0.7

0.1

0.0

0.2

0.0

10.8

57.6

95.0
56.0

22,7

8

60.0

32.1

7.8

5.8

94.2

$\begin{array}{ccc}5.9 & 9.7 & 100 \\ 7,0 & 9,6 & 100 \\ 5 & 7 & -\end{array}$

$\begin{array}{lll}69.5 & 45.9 & 58.5\end{array}$

$\begin{array}{lll}21.8 & 40.9 & 33.7\end{array}$

$\begin{array}{lll}0.1 & 7.3 & 6.8\end{array}$

${ }^{1}$ Pourcentages, ${ }^{2}$ Rang, ${ }^{3}$ Nombre d'individus, ${ }^{4}$ Années

Sources : INSD (1996), Lachaud (1997)

Dans un deuxième temps, les profils de pauvreté de chaque groupe de ménages ont été obtenus à partir de la ligne de pauvreté établie par 
l'INSD (1996). Elle est de 41099 Fcfa par adulte et par an et a été calculée sur la base d'un besoin quotidien alimentaire estimé à 2283 calories, auquel s'ajoutent des dépenses non alimentaires représentant $47 \%$ du budget total. Les évaluations monétaires de ces dépenses ont été faites au prix d'octobre 1994. Les indicateurs F-G-T obtenus sont présentés dans le tableau 2. Ils ont été calculés par équivalent adulte, à partir du nombre d'individus du ménage et d'une échelle d'équivalence qui prend en compte le moindre coût relatif des enfants et la présence éventuelle d'économies d'échelle ${ }^{19}$. Ils confirment l'étendue du phénomène au Burkina Faso et l'extrême diversité des situations parmi les ménages. La pauvreté concerne ainsi près du tiers des ménages. Elle touche en priorité les ménages agricoles et notamment les agriculteurs de subsistance. À l'inverse, les groupes de salariés et les indépendants non agricoles évolutifs sont les moins concernés par le phénomène.

Tableau 2: Indicateurs $F-G-T^{1}$ de chaque groupe de ménage

\begin{tabular}{cccc}
\hline Indices $P_{\alpha}^{(3)}$ & Incidence & Profondeur & Intensité \\
& $\left(\boldsymbol{P}_{\mathbf{0}}\right)$ & $\left(\boldsymbol{P}_{\mathbf{1}}\right)$ & $\left(\boldsymbol{P}_{\mathbf{2}}\right)$ \\
\hline
\end{tabular}

Ligne de pauvreté : $\mathrm{LP}=41099 \mathrm{FCFA}$

Type de ménage

Salariés protégés

0.7

0.1

0.0

Salariés non protégés

4.2

1.2

0.5

Indépendants non agricoles évolutifs

1.5

0.1

0.0

Indépendants non agricoles involutifs

7.8

2.3

1.5

Agriculteurs progressifs

42.1

12.0

4.8

Agriculteurs de subsistance

44.4

14.0

6.0

Éleveurs

42.1

10.6

3.8

Autres

27.9

6.2

2.3

Ensemble

32.4

10.0

4.3

1 Indicateurs de la classe Foster-Greer-Thorbecke (1984). ${ }^{2}$ Tous les résultats ont été multipliés par 100. ${ }^{3} P_{\alpha}=\int^{q}[(l p-x) / l p]^{\alpha} d x$ où $l p$ est la ligne de pauvreté, $x$ le niveau de consommation et q le nombre de pauvres dans la population totale

Sources : Calculs propres d'après l'Enquête Prioritaire 1994-1995 et Lachaud (1997)

Le graphique 1 montre enfin la répartition intra-groupe des dépenses de consommation de chaque groupe de ménages. Les positions par rapport à la ligne de pauvreté confirment les différences constatées entre

19 Deaton (1997). 
les ménages et notamment la précarité des ménages ruraux. Compte tenu de nos choix méthodologiques, la forme de chacune de ces distributions sera considérée comme invariante entre deux chocs exogènes et ne pourra subir que des déplacements homothétiques.

Graphique 1 : Fonction de distribution des dépenses de consommation et situation face à la pauvreté de chaque groupe de ménages

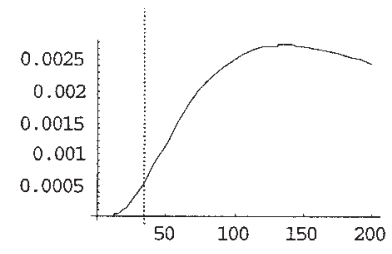

Salariés protégés

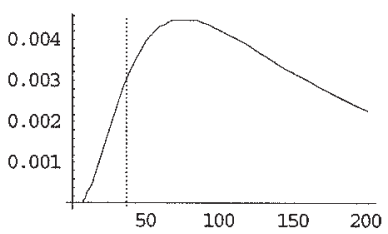

Salariés non protégés

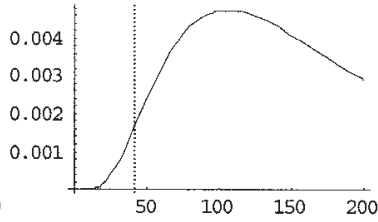

Indépendants évolutifs

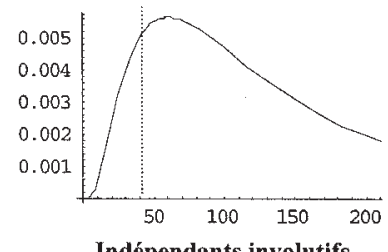

Indépendants involutifs

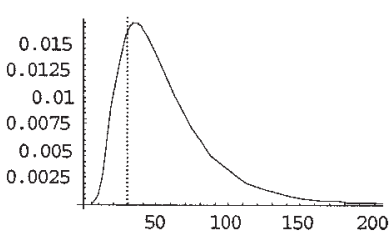

Eleveurs

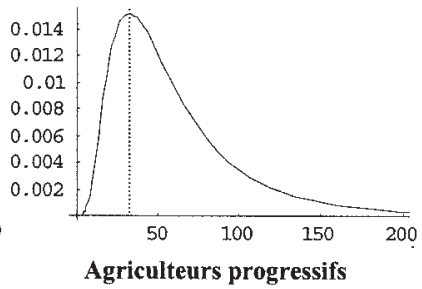

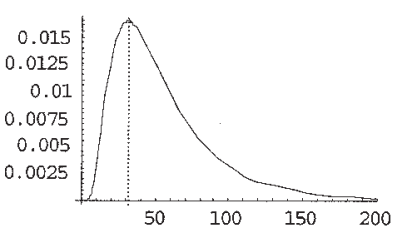

Agriculteurs de subsistance

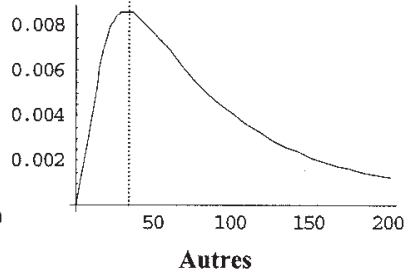

Autres

\section{CONSTRUCTION D'UN MODĖLE EGC DESTINÉ À L'ANALYSE DE LA PAUVRETÉ}

La structure générale du modèle est relativement standard et respecte les principes généraux de la modélisation EGC appliquée aux économies en développement ${ }^{20}$. L'ensemble des variables et paramètres est présenté en annexe. L'offre est assurée par cinq branches dont le choix a été contraint par la nomenclature des secteurs de l'enquête prioritaire. Du

20 Sur ces différentes modalités voir Dervis, De Melo et Robinson (1982). 
coté des branches marchandes (indicées $i$ ou $j$ ), on distingue un secteur « Primaire (1)», composé des activités agricoles, élevage, pêche, chasse et sylviculture, un secteur « Secondaire (2)», regroupant l'ensemble des activités à caractère industriel, un secteur «Bâtiments et Travaux Publics (3) », et un secteur «Tertiaire (4)». La branche «Services non marchands », (indicée $n m$ ) comprend la totalité des administrations publiques et des institutions privées sans but lucratif. Douze types d'agents sont présents dans le modèle: les huit groupes de ménages (indicés par $M_{h}$ ), les entreprises (indicées par E), l'État (indicé par G) et le reste du monde (indicé par F). Il y a enfin quatre types de produits domestiques ou exportés $(i=j=4)$, et cinq types de produits importés $(m=5)$.

\subsection{Production et demande de facteurs}

Chaque branche marchande produit avec une fonction à élasticité de substitution constante (équations 1 à 4) combinant du travail et du capital supposé fixe à court terme. Leur demande de travail se déduit de l'hypothèse de maximisation de profit (équations 5 à 8).

$$
\begin{array}{cc}
X_{i}=A_{i}^{p} \cdot\left[\alpha_{i}^{p} \cdot L D_{i}^{-\mu_{i}^{p}}+\left(1-\alpha_{i}^{p}\right) \cdot \bar{K}_{i}^{-\mu_{i}^{p}}\right]^{-\frac{1}{\mu_{i}^{p}}} & 1-4 \\
\frac{L D_{i}}{X_{i}}=\left[\frac{P V A_{i} \cdot \alpha_{i}^{p}}{\left(A_{i}^{p}\right)^{\mu_{i}^{p}} \cdot w_{i}}\right]^{\sigma_{i}^{p}} & 5-8
\end{array}
$$

Le facteur travail se compose de travailleurs protégés, dont le nombre est supposé fixé à court terme dans chaque branche, et de travailleurs non protégés (équations 9 à 12). Le coût unitaire du travail dans la branche dépend des masses salariales respectives de ces deux types de main-d'oeuvre (équations 12 à 16).

$$
\begin{array}{cc}
L D_{i}=L n p_{i}^{D}+\overline{L p_{i}^{D}} & 9-12 \\
w_{i}=\frac{w n p \cdot L n p_{i}^{D}+\overline{w p} \cdot \overline{L p_{i}^{D}}}{L D_{i}} & 12-16
\end{array}
$$

La branche non marchande est spécifiée comme un simple système de demande. La totalité de sa valeur ajoutée est redistribuée aux fonctionnaires (essentiellement des travailleurs protégés) dont le nombre est supposé fixe à court terme.

$$
\overline{w p} \cdot \overline{L p_{n m}^{D}}=P V A_{n m} \cdot X_{n m}
$$




\subsection{Biens domestiques, exportations et importations}

Les biens domestiques sont exclusivement destinés aux marchés du Burkina Faso et, compte tenu de la nature de l'appareil productif de ce pays, sont considérés comme non substituables avec les biens importés. En revanche, une fonction à élasticité de transformation constante (CET) est spécifiée entre les biens domestiques et les exportations, essentiellement composées de produits primaires (équations 18 à 21 et 22 à 25).

$$
\begin{array}{cc}
X_{i}=A_{i}^{t} \cdot\left[\alpha_{i}^{t} \cdot D S_{i}^{-\mu_{i}^{t}}+\left(1-\alpha_{i}^{t}\right) \cdot E_{i}^{-\mu_{i}^{t}}\right]^{-\frac{1}{\mu_{i}^{t}}} & 18-21 \\
\frac{D S_{i}}{E_{i}}=\left[\frac{\alpha_{i}^{t}}{\left(1-\alpha_{i}^{t}\right)}\right]^{\sigma_{i}^{t}} \cdot\left[\frac{P D_{i}}{P E_{i}}\right]^{\sigma_{i}^{l}} & 22-25
\end{array}
$$

\subsection{Système de prix}

Selon l'hypothèse du petit pays, le prix des biens exportés est donné par les cours mondiaux (équations 26 à 29). La même règle est adoptée pour le prix des biens importés auquel s'ajoute une taxe forfaitaire à l'importation (équations 30 à 34).

$$
\begin{aligned}
& P E_{i}=E R \cdot \overline{P W E}_{i} \\
& 26-29 \\
& P M_{m}=E R \cdot \overline{P W M_{m}} \cdot\left(t m_{m}+1\right) \\
& 30-34
\end{aligned}
$$

Le prix à la production des branches marchandes exportatrices se déduit de la fonction CET.

$$
P X_{i}=\frac{P D_{i} \cdot D S_{i}+P E_{i} \cdot E_{i}}{X_{i}}
$$

Dans tous les secteurs, le prix à la valeur ajoutée dépend du prix à la production (diminué d'un impôt pour les branches marchandes) et du prix des consommations intermédiaires domestiques et importées.

$$
\begin{gathered}
P V A_{j}=P X_{j} \cdot\left(1-t x_{j}\right)-\sum_{i} P D_{i} \cdot a d_{i, j}-\sum_{m} P M_{m} \cdot a m_{m, j} 39-42 \\
P V A_{n m}=P_{n m}-\sum_{i} P D_{i} \cdot a d_{i, n m}-\sum_{m} P M_{m} \cdot a m_{m, n m}
\end{gathered}
$$

Pour pouvoir caractériser le nouveau profil de pauvreté des groupes de ménages après simulation d'un choc, une ligne de pauvreté est introduite 
dans le modèle. Son niveau est déterminé de manière endogène par le système de prix caractérisant chaque équilibre ${ }^{21}$.

$$
L P=\sum_{i} \beta_{i} \cdot P D_{i}+\sum_{m} \beta_{m} \cdot P M_{m}
$$

\subsection{Dotations en capital des ménages, formation et utilisation du revenu des agents}

Conformément aux options méthodologiques retenues, le niveau de revenu de chaque type de ménage découle de leurs dotations initiales en actifs, révélatrices de leur degré de vulnérabilité (équations 45 à 52). Leur dotation en capital physique $\left(\gamma_{h}\right)$ détermine leur accès aux revenus du capital. Leur dotation en capital humain $\left(l_{h, n p}\right.$ et $\left.l_{h, p}\right)$, ici révélée par leur degré « d'employabilité », détermine leur accès aux revenus du travail protégé (privé ou public) ou non protégé. Leur dotation en capital social est supposée déterminer leur accès aux revenus de transferts ${ }^{22}$. Deux types de capital social sont distingués à ce niveau $\left(t_{g h}\right.$ et $\left.t_{f h}\right)$. Le premier concerne la capacité à bénéficier des transferts publics et le second la capacité à bénéficier des transferts de fonds des émigrés vers leurs familles restées au Burkina Faso ${ }^{23}$.

$$
\begin{array}{r}
Y M_{h}=l_{h, n p} \cdot w n p \cdot \overline{L n p^{S}}+l_{h, p} \cdot w p \cdot L p^{S}+r_{h, a g} \cdot R K a g \\
+r_{h, n a g} \cdot R K n a g+t g_{h} \cdot \overline{T G M}+t f_{h} \cdot E R \cdot \overline{T F M} \\
R K a g=P V A_{1} \cdot X_{1}-w_{1} \cdot L D_{1} \\
R K n a g=\sum_{i \neq 1}\left(P V A_{i} \cdot X_{i}-w_{i} \cdot L D_{i}\right)
\end{array}
$$

Chaque ménage verse des impôts et épargne une partie de son revenu disponible selon une propension fixe (équations 55 à 62). Il utilise le reste pour la consommation de produits non marchands et de produits marchands domestiques ou importés (équations 71 à 78). La consommation de ces derniers est supposée fixée en volume pour chaque ménage

$$
S M_{h}=s m_{h} \cdot(1-\text { tym }) \cdot Y M_{h} \quad 55-62
$$

21 Les coefficients de pondération de la ligne de pauvreté ont été choisis conformément aux options méthodologiques retenues par l'INSD (1996).

22 Lachaud (1999).

23 Le montant de ces transferts de fonds est en effet loin d'être négligeable au Burkina Faso (près de $14 \%$ du revenu total de ménages) et il est en grande partie mobilisé par les agriculteurs de subsistance. 


$$
\begin{array}{cc}
C F_{h}=Y M_{h}-S M_{h} & 63-70 \\
C F_{h}=C F D_{h}+\sum_{m} P M_{m} \cdot C M Q_{m, h}+\overline{C M N_{h}} & 71-78
\end{array}
$$

Le revenu de l'État correspond aux différents impôts (équation 79). L'épargne publique se détermine une fois les dépenses publiques effectuées. Ces dernières (transferts vers les ménages et le reste du monde, consommation de services non marchands et investissements publics) sont supposées fixées en valeur (équation 80).

$$
\begin{array}{r}
Y G=\sum_{h} t_{y m_{h}} \cdot Y M_{h}+\text { tye } \cdot Y E+\sum_{i} t x_{i} \cdot P X_{i} \cdot X_{i}+\sum_{m} t m_{m} \cdot P M_{m} \cdot M_{m} \\
S G=Y G-\overline{T G M}-\overline{T G F}-\overline{C N M_{G}}-\overline{I G}
\end{array}
$$

Les entreprises reçoivent la part non distribuée des revenus du capital (équation 81). Elles épargnent la totalité de leur revenu disponible après le paiement des impôts et transferts vers le reste du monde (équation $82)$.

$$
\begin{aligned}
Y E & =r e \cdot(R K a g+R K n a g) \\
S E & =(1-t y e) \cdot Y E-\overline{T E F}
\end{aligned}
$$

\subsection{Demande de biens et services}

Les dépenses de consommation en biens domestiques pour chaque ménage prennent la forme d'un système de dépenses linéaire (équations 83 à 114). La demande intermédiaire en volume se déduit de la matrice des coefficients techniques (équations 115 à 118). Les demandes d'investissement public et privé sont déterminées en volume à partir d'une clé de répartition exogène (équations 119 à 122 et 123 à 126).

$$
\begin{array}{rr}
P D_{i} \cdot C D Q_{i, h}=c \min _{i, h} \cdot P D_{i}+p m c_{i, h} \cdot\left[C F D_{h}-\sum_{j} c \min _{j, h} \cdot P D_{j}\right. \\
D I Q_{i}=\sum_{j} a d_{i, j} \cdot X_{j}+a d_{i, n m} \cdot X_{n m} & 83-114 \\
P D_{i} \cdot I G D Q_{i}=t g d_{i} \cdot I \bar{G} & 115-118 \\
P D_{i} \cdot I P D Q_{i}=t p d_{i} \cdot I P & 119-122
\end{array}
$$

La demande de produits importés par le ménage $h$ se fait selon des propensions à consommer exogènes (équations 127 à 166). Les demandes 
en volume d'investissement privé et public sont également déterminées à partir d'une clé de répartition exogène (équations 175 à 179 et 180 à 184). La demande intermédiaire découle des contraintes techniques de production (équations 185 à 189).

$$
\begin{array}{rr}
P M_{m} \cdot C M Q_{m, h}=\phi_{m, h} \cdot C F M_{h} & 127-166 \\
C F M_{h}=\sum_{m} P M_{m} \cdot C M Q_{m, h} & 167-174 \\
P M_{m} \cdot I G M Q_{m}=t g m_{m} \cdot I \bar{G} & 175-179 \\
P M_{m} \cdot I P M Q_{m}=t p m_{m} \cdot I P & 180-184 \\
M I Q_{m}=\sum_{j} a m_{m, j} \cdot X_{j}+a m_{m, n m} \cdot X_{n m} & 185-189
\end{array}
$$

\section{6 Équilibre sur les marchés et mode de bouclage}

Sur chaque marché de biens et services domestiques ou importés, on retrouve l'égalité entre l'offre et la demande. De même, la demande totale de services non marchands est égale à l'offre de la branche.

$$
\begin{array}{cr}
D S_{i}=\sum_{h} C D Q_{i, h}+D I Q_{i}+I G D Q_{i}+I P D Q_{i} & 190-193 \\
M_{m}=\sum_{h} C M Q_{m, h}+M I Q_{m}+I G M Q_{m}+I P M Q_{m} & 194-198 \\
P n m \cdot X n m=\sum_{h} \overline{C N M_{h}}+\overline{C N M_{G}} & 199
\end{array}
$$

Conformément au choix de désagrégation des ménages, le marché du travail est segmenté entre les salariés non protégés et les salariés protégés. Les deux segments sont en équilibre de plein emploi (équations 200 et 201$)^{24}$. Sur le segment protégé, le salaire est supposé rigide et la main-d'œuvre est protégée des licenciements. On suppose donc que les entreprises profitent de la flexibilité des salaires et de l'embauche de la main-d'œuvre salariée non protégée pour satisfaire leur demande de travail.

$$
\overline{L n p^{S}}=\sum_{i} L n p_{i}
$$

24 Le niveau de l'emploi public est supposé exogène. 


$$
L p^{S}=\sum_{i} \overline{L p_{i}}+\overline{L p_{n m}}
$$

Les deux dernières conditions d'équilibre concernent la balance des paiements et l'égalité entre l'épargne et l'investissement ${ }^{25}$.

$$
\begin{array}{cr}
\overline{S F}=\sum_{m} E R \cdot P W M_{m} \cdot M_{m}-\sum_{i} P E_{i} \cdot E_{i}+\overline{T G F}+\overline{T E F}-E R \cdot \overline{T F M} \\
I P+\overline{I G}=\sum_{h} S M_{h}+S E+S G+\overline{S F}
\end{array}
$$

Le taux de change est posé comme numéraire. Les variations de statique comparative constatées dans les simulations devront donc être interprétées en fonction de ce numéraire.

$$
E R=1
$$

\section{4 ÉLABORATION D'UNE MATRICE DE COMPTABILITÉ SOCIALE POUR LE BURKINA FASO}

Compte tenu des faiblesses de la Comptabilité Nationale burkinabé et du contenu spécifique de cette étude, nous avons choisi d'élaborer notre propre Matrice de Comptabilité Sociale (MCS) pour assurer un support empirique au modèle EGC. Elle est construite à partir des données macroéconomiques estimées pour le Burkina Faso dans le cadre de l'Instrument Automatisé de Prévision (IAP) ${ }^{26}$ pour l'année 1995, ainsi que des données complémentaires sur les ménages fournies par l'enquête prioritaire 1994-1995. Toute la difficulté consiste alors à concilier ces deux sources de données distinctes.

Dans un premier temps, les informations sur les relations intersectorielles, les processus de production, la nature, l'origine et la destination

25 Conformément à la logique Néo-Classique qui sous-tend le modèle, le niveau total de l'investissement dépend du niveau préalable de l'épargne dans l'économie.

26 Ministère de l'Économie, des Finances et du Plan du Burkina Faso, GTZ (1995). L'IAP est un programme de gestion macroéconomique prévisionnelle dont le Burkina Faso s'est doté depuis 1992 en collaboration avec la Coopération Technique Allemande et avec le soutien de l'Union Européenne. C'est un modèle «quasi-comptable» de type récursif permettant des projections des principaux agrégats macroéconomiques. Il n'a pas de caractère officiel et ne prétend pas être un instrument de Comptabilité Nationale mais fournit cependant des ordres de grandeurs fiables compte tenu de la rigueur méthodologique qui a guidé son élaboration. Les données initiales de l'IAP ont été extrapolées à partir du dernier TES officiel de 1985. 
des différents produits sont extraites du Tableau Entrées-Sorties (TES) estimé par l'IAP, dont les onze branches ont été réduites à cinq conformément à la nomenclature de l'enquête prioritaire.

Dans un deuxième temps, les différents sous-groupes de ménages sont incorporés dans la MCS en fonction de leurs sources de revenus respectives. Compte tenu des écarts observés entre les deux sources de données et de la nécessité de respecter l'équilibre du TES entre la production, les revenus et la demande, l'idée directrice est de privilégier systématiquement les données macroéconomiques de l'IAP et de leur appliquer les clés de désagrégation dégagées lors de l'analyse des données de l'enquête prioritaire. Malgré cette précaution, il apparaît que les revenus du capital sont largement sous-estimés dans cette dernière enquête ${ }^{27}$ et qu'un réajustement supplémentaire s'avère nécessaire pour maintenir la cohérence du TES entre les parts respectives de revenu du travail et de revenu du capital ${ }^{28}$. Au final, la distribution inter-groupes des revenus est respectée et le revenu moyen estimé de chaque sous-groupe est réparti entre les six sources potentielles pouvant contribuer à sa formation. Le partage des différentes sources de revenus entre les ménages permet de déduire leurs dotations respectives en actifs et donc leur degré de vulnérabilité. Elles sont présentées dans le tableau 3.

Dans un troisième temps, la consommation totale est désagrégée entre les différents ménages en respectant la cohérence du TES. Là encore, certains ajustements s'avèrent nécessaires car les clés de répartition des dépenses de consommation, déduites de l'enquête prioritaire, révèlent une large surestimation des différentes propensions à consommer ${ }^{29}$, très certainement liée à la prise en compte de l'autoconsommation. Pour contourner ce problème, il a été supposé une part d'autoconsommation décroissante avec le niveau de revenu ${ }^{30}$.

La construction de la MCS s'achève enfin en incluant certaines informations complémentaires tirées de l'IAP et en soldant le compte de chaque agent (les Ménages, les Entreprises, l'État et le Reste du monde) par leur épargne dans le compte de capital ${ }^{31}$.

27 Nous pouvions nous y attendre a priori car si l'information sur les salaires est relativement fiable au Burkina Faso, il n'en va pas de même pour les revenus du capital. De plus, dans l'enquête prioritaire, l'information sur les revenus globaux n'est pas disponible pour $7.8 \%$ des ménages.

28 Il a été supposé un même «taux de non-déclaration » des revenus du capital pour tous les agents ménages.

29 Cela se traduit notamment par des taux d'épargne négatifs pour les ménages les plus défavorisés.

30 En l'absence d'information dans ce domaine, il est impossible de distinguer les parts respectives de la consommation en produits importés et locaux. La même clé de répartition exogène (tirée des données de l'IAP) a été arbitrairement appliquée à tous les types de ménages.

31 La MCS finale ainsi obtenue est disponible auprès de l'auteur. 
Tableau 3: Dotations respectives en capital économique et social des différents ménages au Burkina Faso
Type de capital Employabilité
Capital physique
Capital social

$\begin{array}{lcccc}\text { Salariat } & \text { Salariat } & \text { Capital Capital } & \text { Transferts Transferts } \\ \text { protégé non protégé agricole non agricole } & \text { publics } & \text { privés }\end{array}$

Type de Ménage

\begin{tabular}{lcccccc} 
Salariés protégés & 100,0 & 0,0 & 0,7 & 3,0 & 13,7 & 6,9 \\
Salariés non protégés & 0,0 & 82,2 & 0,7 & 2,0 & 3,8 & 3,0 \\
$\begin{array}{l}\text { Indépendants non } \\
\text { agricoles évolutifs }\end{array}$ & 0,0 & 2,1 & 1,3 & 15,8 & 1,6 & 0,8 \\
$\begin{array}{l}\text { Indépendants non } \\
\text { agricoles involutifs }\end{array}$ & 0,0 & 5,0 & 2,8 & 32,3 & 18,3 & 8,5 \\
Agriculteurs progressifs & 0,0 & 1,0 & 25,9 & 9,4 & 1,3 & 6,1 \\
Agriculteurs de subsistance & 0,0 & 3,7 & 41,2 & 25,9 & 16,3 & 38,1 \\
Éleveurs & 0,0 & 0,4 & 19,5 & 4,5 & 0,6 & 4,1 \\
Autres & 0,0 & 5,5 & 7,9 & 7,1 & 44,6 & 32,4 \\
\hline Ensemble & 100,0 & 100,0 & 100,0 & 100,0 & 100,0 & 100,0 \\
\hline
\end{tabular}

Sources : calculs propres d'après Lachaud (1997, 1999), MEFP-GTZ(1995) et INSD(1996)

\section{SIMULATIONS DE L'IMPACT DE CHOCS MACROÉCONOMIQUES SUR LA PAUVRETÉ DANS L'ÉCONOMIE BURKINABÉ}

Une fois le modèle calibré sur les données de la MCS, censée caractériser l'équilibre général de référence, il est possible de simuler numériquement le comportement de l'économie burkinabé face à des chocs exogènes. Les raisonnements se font en statique comparative. Chaque nouvel équilibre général, c'est-à-dire chaque nouveau système de prix d'équilibre, définit une nouvelle ligne de pauvreté dans l'économie et un nouveau niveau de consommation moyen pour chaque ménage représentatif. En supposant les distributions intra-groupe des dépenses de consommation constantes, il est possible de caractériser la situation face à la pauvreté de chaque ménage pris individuellement, puis d'évaluer les effets des différents chocs en termes d'incidence, de profondeur et d'intensité sur la pauvreté de chaque groupe de ménages. Pour tenter de minimiser encore plus l'hypothèse de la constance des distributions intra-groupe, nous avons décidé de raccourcir l'horizon de l'analyse au court terme. Cette option se révèle alors essentiellement dans le choix des simulations 
effectuées qui écarte les politiques ou les chocs macroéconomiques impliquant des mouvements longs dans les mécanismes induits. Les résultats obtenus doivent évidemment être interprétés avec prudence compte tenu des approximations nécessaires pour construire la base de données et des limites méthodologiques inhérentes à l'approche utilisée. C'est donc avant tout le sens des variations des mécanismes induits qu'il est important d'observer plutôt que leur niveau absolu.

\subsection{Chocs affectant les échanges extérieurs}

Les trois premières mesures envisagées concernent des chocs influençant les échanges extérieurs. Les résultats obtenus sont présentés dans le tableau 4.

La première simulation consiste en une réduction de $20 \%$ des droits de douanes. Compte tenu de l'importance des recettes fiscales liées aux importations et de la nécessité pour l'État de maîtriser son déficit budgétaire, on suppose également que cette mesure s'accompagne d'une augmentation de la pression fiscale sur les entreprises et sur les ménages. Cette mesure peut alors s'inscrire dans la volonté d'ouverture affichée par le Burkina Faso ces dernières années en matière d'intégration régionale vers l'UMOA ou la CDEAO ou encore dans le processus de déréglementation initié par les PAS. Si cette mesure a un impact globalement positif sur la consommation et la pauvreté des ménages, il reste toutefois limité. Le caractère non substituable des importations, et donc leur moindre sensibilité aux variations de prix dans le modèle, ainsi que la faible part des importations dans la consommation finale des ménages est certainement un élément d'explication. Du coté de l'offre, la baisse du prix des importations a un effet immédiatement positif sur la valeur ajoutée de chaque branche compte tenu de la présence d'intrants importés dans les processus de production. Certains groupes de ménages, tels les salariés protégés, les salariés non protégés ou les indépendants non agricoles évolutifs, à l'origine faiblement concernés par le phénomène de pauvreté, ne voient pas leur situation changer. Les autres groupes de ménages, en revanche, voient leur situation s'améliorer légèrement.

La seconde simulation concerne une augmentation exogène de $15 \%$ du cours mondial des exportations agricoles. Le choix de cette simulation se justifie par la forte spécialisation de l'économie burkinabé autour de quelques produits d'exportation à faible valeur ajoutée, qui témoigne de sa dépendance par rapport aux cours mondiaux des produits agricoles ou primaires, et par l'importance des revenus agricoles dans la forma- 
Tableau 4 : Indicateurs de pauvreté après simulation de chocs exogènes sur les échanges extérieurs

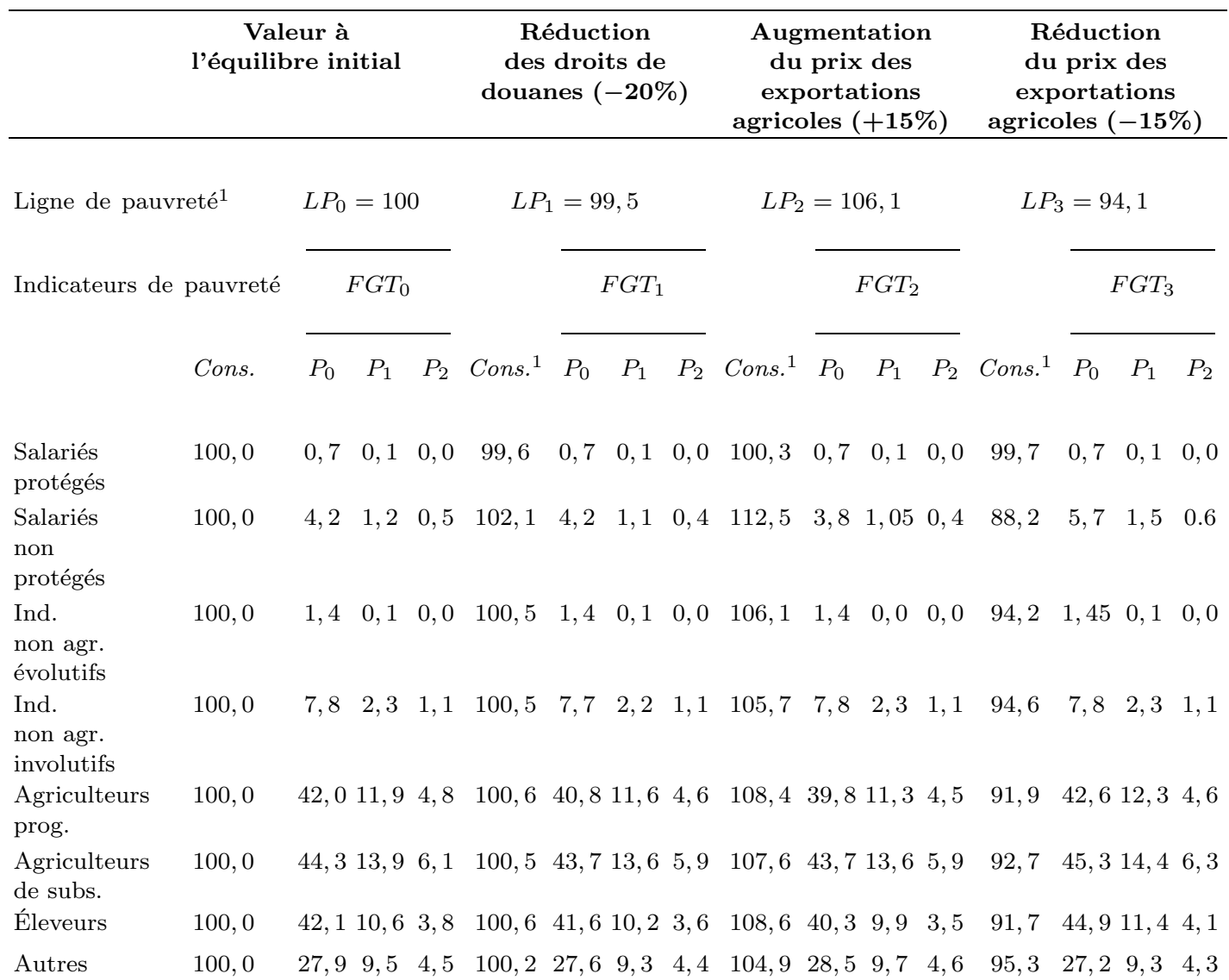

Ensemble

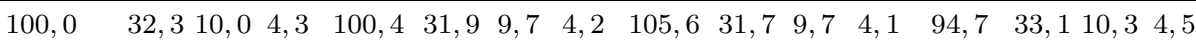

1 41099FCFA à l'équilibre initial

Sources: Calculs propres

tion des niveaux de vie des ménages. Une telle augmentation, que les autorités ne maîtrisent pas, peut alors s'interpréter comme un rétablissement des marchés agricoles ou comme un effort de la part d'institutions internationales, telles que l'Union économique européenne par exemple, pour garantir des prix planchers aux pays en développement. Deux effets contraires en termes de pauvreté s'additionnent sans toutefois s'annuler. Dans un premier temps, on assiste à une nette augmentation de la ligne de pauvreté. Dans un second temps, la consommation totale des 
ménages augmente fortement à la suite de la hausse de leur pouvoir d'achat. Au total, sur l'ensemble des ménages, le second effet l'emporte sur le premier et les conséquences de ce choc apparaissent clairement positives en termes de réduction de la pauvreté. Lorsque l'on analyse la situation des différents types de ménages, on s'aperçoit que ce sont les ménages agricoles qui bénéficient largement de cette augmentation des prix et qui voient leurs indicateurs de pauvreté s'améliorer. On pouvait s'y attendre compte tenu de l'importance des revenus agricoles dans le mode de formation des revenus. Comme ces ménages constituent la majorité de la population burkinabé, l'effet d' entraînement de cette mesure est large. Les autres grands gagnants sont alors les ménages salariés non protégés qui bénéficient de cette reprise générale de l'activité économique qui se répercute sur le marché du travail à travers l'augmentation de leur taux de salaire.

À ce stade de l'analyse, compte tenu de l'importance des effets induits par le choc précédent, il nous a semblé intéressant de simuler les effets d'une diminution du prix des exportations des produits agricoles. Dans les années quatre-vingt, la chute continue du prix du coton a par exemple largement entamé les réserves de change du Burkina Faso et réduit ses potentialités de croissance. Raffinot (1997) souligne qu'elle a également eu une répercussion sur les finances publiques puisque l'État burkinabé a été conduit à déconnecter le prix domestique du prix international en subventionnant largement la filière. L'effet est nettement négatif malgré la réduction de la ligne de pauvreté liée au contexte désinflationniste. L'incidence de la pauvreté augmente de près de $2,5 \%$ sur l'ensemble du pays. Les ménages les plus concernés restent évidemment les ménages agricoles et surtout les ménages salariés non protégés. Ce constat révèle ici la forte vulnérabilité de ces derniers face aux ajustements macroéconomiques du pays.

\subsection{Simulations de politiques publiques}

Les trois mesures suivantes concernent l'impact sur la pauvreté de mesures de politiques économiques. Les résultats obtenus sont présentés dans le tableau 5.

La première politique envisagée est une augmentation de $20 \%$ des investissements publics. Compte tenu de la faible capacité budgétaire de l'État burkinabé et de la nécessité de pratiquer une relative rigueur en matière de finances publiques, il est également supposé que cette mesure est financée en totalité par l'épargne étrangère. Les effets en termes de pauvreté sont clairement positifs pour l'ensemble des ménages malgré 
l'augmentation des prix liée à l'augmentation de la demande. Tous les groupes de ménages voient leur situation s'améliorer à part les ménages salariés dont le pouvoir d'achat s'érode sous l'effet de l'inflation et de la constance du taux de salaire spécifié dans le modèle. Un tel scénario n'apparaît donc valide qu'à court terme, car il est à prévoir que, compte tenu de la protection dont ils bénéficient, ces derniers verront rapidement leur salaire s'indexer sur la hausse des prix.

Tableau 5: Indicateurs de pauvreté après simulation de politiques publiques

\begin{tabular}{|c|c|c|c|c|c|c|c|c|c|c|c|c|c|c|c|c|}
\hline & \multicolumn{4}{|c|}{$\begin{array}{c}\text { Valeur à } \\
\text { l'équilibre initial }\end{array}$} & \multicolumn{4}{|c|}{$\begin{array}{c}\text { Augmentation } \\
\text { des } \\
\text { investissements } \\
\text { publics }(+20 \%)\end{array}$} & \multicolumn{4}{|c|}{$\begin{array}{l}\text { Réduction des } \\
\text { transferts vers } \\
\text { les ménages } \\
(-20 \%)\end{array}$} & \multicolumn{4}{|c|}{$\begin{array}{c}\text { Réduction des } \\
\text { transferts de } \\
\text { fonds vers les } \\
\text { ménages }(-15 \%)\end{array}$} \\
\hline \multicolumn{2}{|c|}{ Ligne de pauvreté ${ }^{1}$} & \multicolumn{3}{|c|}{$L P_{0}=100$} & \multicolumn{4}{|c|}{$L P_{4}=109,9$} & \multicolumn{4}{|c|}{$L P_{5}=97,6$} & \multicolumn{4}{|c|}{$L P_{6}=90,8$} \\
\hline \multicolumn{2}{|c|}{ Indicateurs de pauvreté } & \multicolumn{3}{|c|}{$F G T_{0}$} & \multicolumn{4}{|c|}{$F G T_{4}$} & \multicolumn{4}{|c|}{$F G T_{5}$} & \multicolumn{4}{|c|}{$F G T_{6}$} \\
\hline & Cons. & $P_{0}$ & $P_{1}$ & $P_{2}$ & Cons. $^{1}$ & $P_{0}$ & $P_{1}$ & $P_{2}$ & Cons. $^{1}$ & $P_{0}$ & $P_{1}$ & $P_{2}$ & Cons. $^{1}$ & $P_{0}$ & $P_{1}$ & $P_{2}$ \\
\hline $\begin{array}{l}\text { Salariés } \\
\text { protégés }\end{array}$ & 100,0 & 0,7 & 0,1 & 0,0 & 100,7 & 1,0 & 0,2 & 0,1 & 99,4 & 0,7 & 0,1 & 0,0 & 99,1 & 0,7 & 0,1 & 0,0 \\
\hline $\begin{array}{l}\text { Salariés non } \\
\text { protégés }\end{array}$ & 100,0 & 4,2 & 1,2 & 0,5 & 137,5 & 2,6 & 0,7 & 0,2 & 94,7 & 4,9 & 1,3 & 0,5 & 77,8 & 7,7 & 1,9 & 0,8 \\
\hline $\begin{array}{l}\text { Ind. } \\
\text { non agr. } \\
\text { évolutifs }\end{array}$ & 100,0 & 1,4 & 0,1 & 0,0 & 115,1 & 1,4 & 0,0 & 0,0 & 97,5 & 1,4 & 0,1 & 0,0 & 88,9 & 1,4 & 0,1 & 0,0 \\
\hline $\begin{array}{l}\text { Ind. } \\
\text { non agr. } \\
\text { involutifs }\end{array}$ & 100,0 & 7,8 & 2,3 & 1,1 & 114,0 & 7,2 & 2,1 & 1,0 & 96,5 & 8,1 & 2,3 & 1,1 & 89,0 & 8,1 & 2,4 & 1,1 \\
\hline $\begin{array}{l}\text { Agriculteurs } \\
\text { prog. }\end{array}$ & 100,0 & 42,0 & 11,9 & 4,8 & 112,6 & 39,8 & 11,2 & 4,4 & 97,1 & 42,3 & 12,1 & 4,9 & 88,7 & 43,0 & 12,6 & 5,1 \\
\hline $\begin{array}{l}\text { Agriculteurs } \\
\text { de subs. }\end{array}$ & 100,0 & 44,3 & 13,9 & 6,1 & 112,3 & 43,1 & 13,3 & 5,7 & 96,9 & 44,9 & 14,2 & 6,2 & 88,0 & 46,31 & 14,9 & 6,6 \\
\hline Éleveurs & 100,0 & 42,1 & 10,6 & 3,8 & 112,3 & 40,3 & 9,9 & 3,5 & 97,0 & 42,5 & 10,8 & 3,9 & 88,7 & 44,71 & 11,3 & 4,1 \\
\hline Autres & 100,0 & 27,9 & 9,5 & 4,5 & 109,0 & 28,4 & 9,6 & 4,6 & 94,2 & 29,3 & 10,2 & 4,8 & 88,7 & 29,3 & 9,9 & 4,7 \\
\hline Ensemble & 100,0 & 32,3 & 10,0 & 4,3 & 111,8 & 31,4 & 9,5 & 4,1 & 97,0 & 32,9 & 10,2 & 4,4 & 90,2 & 33,91 & 10,6 & 4,6 \\
\hline
\end{tabular}


La seconde politique concerne au contraire une réduction des transferts publics vers les ménages. Celle-ci peut être interprétée dans la continuité des mesures d'austérité amorcées à partir du début des années quatre-vingt-dix avec la mise en place des PAS. Comme on pouvait s'y attendre, l'effet d'une telle mesure est largement négatif sur l'ensemble des ménages. Tous les revenus régressent ainsi que la consommation et les prix. Les ménages les plus touchés sont sans conteste les salariés non protégés à travers la forte baisse des revenus du travail. Les ménages Autres, dont le capital social est important à travers les transferts publics, voient également leur situation se dégrader. Au final, seuls les ménages les moins vulnérables (les Salariés protégés et les Indépendants évolutifs) semblent résister à cette mesure d'austérité.

La dernière politique concerne une réduction de $15 \%$ des transferts du reste du monde vers les ménages. Nous avons déjà signalé que ces transferts de fonds sont particulièrement importants au Burkina Faso et qu'ils constituent un véritable capital social qui contribue largement à soulager la pauvreté de nombre de ménages, notamment des Agriculteurs de subsistance et des ménages Autres. La réduction de $15 \%$ de ces transferts a ainsi un effet extrêmement négatif en termes de pauvreté. L'ensemble des ménages voit sa situation se dégrader. Seuls les salariés protégés résistent. La situation des Salariés non protégés après le choc confirme, là encore, le caractère extrêmement précaire et vulnérable de leur statut social.

\section{CONCLUSIONS}

L'objectif de cette étude était de contribuer à l'analyse des déterminants de l'ampleur et des mécanismes de diffusion de la pauvreté monétaire au sein de la population des ménages au Burkina Faso. À travers l'utilisation d'un modèle EGC, elle a tenté d'inscrire le phénomène dans la dynamique générale du fonctionnement économique du pays. Elle a également essayé de l'envisager dans ses composantes économiques et sociales en faisant reposer la désagrégation des ménages sur un critère de vulnérabilité pour mettre en avant leurs dotations respectives en capital.

Les simulations numériques ont révélé l'influence des chocs ou des politiques macroéconomiques sur la pauvreté de l'ensemble des ménages et ont montré leurs impacts spécifiques sur les différents sous-groupes distingués. Elles ont par exemple confirmé que l'État burkinabé possédait une certaine marge de manœuvre pour lutter contre la pauvreté glo- 
bale à travers l'augmentation des investissements publics financée par l'endettement extérieur. De même, elles ont révélé que, dans un contexte de forte dépendance des ménages par rapport au reste du monde, certaines politiques macroéconomiques ou sectorielles plus ciblées, telles que la réduction des barrières douanières, la garantie d'un prix plancher pour les exportations agricoles ou encore l'encouragement des transferts de fonds en provenance de l'étranger, pouvaient s'avérer des stratégies efficaces de réduction de la pauvreté au Burkina Faso.

Même s'ils peuvent être sujets à caution et doivent être manipulés avec précaution compte tenu des contraintes méthodologiques qui ont conditionné l'analyse, ces résultats confirment l'opportunité d'envisager les politiques de lutte contre la pauvreté dans une perspective globale, qui tienne compte des conditions macroéconomiques du pays, de ses spécificités sectorielles et des caractéristiques de sa structure sociale. Cette analyse peut donc finalement s'inscrire dans les efforts entrepris ces dernières années pour privilégier une démarche intégrée dans l'élaboration des politiques de lutte contre la pauvreté. Les Cadres Stratégiques de Lutte contre la Pauvreté établis par les pays engagés dans l'Initiative PPTE (Pays Pauvres Très Endettés) ${ }^{32}$ sont à cet égard révélateurs d'une telle approche. Elaborés en concertation avec les différents acteurs du développement du pays, ils s'appuient sur une analyse approfondie de la nature et de la répartition de la pauvreté pour concevoir des stratégies qui permettent d'assurer une cohérence entre les nécessités de l'allègement de la dette, les objectifs des politiques économiques ou sociales et la lutte contre la pauvreté. Le CSLP du Burkina Faso ${ }^{33}$ souligne ainsi qu'une croissance forte et équitable du revenu national est l'une des conditions indispensables pour venir à bout de la pauvreté généralisée. Mais il souligne également qu'elle doit être accompagnée par des mesures structurelles destinées à garantir l'accès des plus pauvres aux services sociaux de base et à élargir leurs opportunités futures en matière d'emplois et d'activités génératrices de revenus. Au total, c'est bien l'approche en termes de dotations en capital qui semble déterminante ici. Cela conforte l'idée que seules des mesures intégrées visant à augmenter la capacité des ménages à faire face au risque de pauvreté apparaissent comme des stratégies durables de lutte contre la pauvreté au Burkina Faso.

32 Voir, par exemple, Joseph (2000) ou Comité du Développement de la Banque Mondiale et du FMI (1999).

33 Ministère de l'Économie et des Finances du Burkina Faso (2000). 


\section{BIBLIOGRAPHIE}

Adelman I., S. Robinson (1979), "Income Distribution Policy : a Computable General Equilibrium Model of South Korea", in ADELMAN I., The selected essays of Irma Adelman. Volume 1. Dynamics and Income Distribution. Economists of the Twentieth Century Series, Aldershot, U.K.

BAnque Mondiale (2001), World Development Report, 2000/2001. Attacking Poverty, Washington, World Bank.

BANQue Mondiale (1990), Rapport sur le développement dans le monde. La Pauvreté, Washington, Banque Mondiale.

Cogneau D. (1997), Le marché du travail, la pauvreté et les inégalités à Antananarivo: une microsimulation en équilibre général, mimeo.

Cogneau D., A.S Robilliard (1999), Income Distribution, Poverty and Growth in Madagascar: Microsimulation in a General Equilibrium Framework, mimeo.

Comité du Développement de la Banque mondiale et du FMI (1999), «Initiative en faveur des pays pauvres très endettés : renforcer le lien entre l'allègement de la dette et la résorption de la pauvreté », Document DC/99-24.

DeAton A., (1997), The Analysis of Household Surveys, Baltimore, The Johns Hopkins University Press.

Decaluwé B., J.C. Dumont, L. Savard (1999), « Mesurer la pauvreté et les inégalités dans un modèle d'Équilibre Général Calculable », GREPE, Working Paper n9920, septembre, Université de Laval, Québec.

Decaluwé B., A. Patry, L. SAVArd (1998), "Income Distribution, Poverty Measure and Trade Shocks : a Computable General Equilibrium Model of a Archetype Developing country", CREFA, Cahier de recherche n98-14.

Decaluwé B., A. Patry, L. SAvard, E. Thorbecke, (1999), "Poverty Analysis within a General Equilibrium Framework", CREFA, Working Paper n9906, June 1999, Université de Laval, Québec.

Dervis K., J. De Melo, S. Robinson (1982), General Equilibrium Models for Development Policy, Cambridge University Press, London.

Foster J.E., J. Greer, E. Thorbecke (1984), «A Class of Decomposable Poverty Measure », Econometrica, vol. 52(3).

INSD (1996), Analyse des résultats de l'enquête prioritaire sur les conditions de vie des ménages, Ouagadougou, février, Institut National de la Statistique et de la Démographie.

Joseph A. (2000), «Résoudre le problème de la dette: de l'initiative PPTE à Cologne », Documents techniques $\mathrm{n}^{\circ} 163$, Centre de Développement de l'OCDE, août 2000.

LACHAUD J.P. (2001), La dynamique de pauvreté au Burkina Faso : éléments d'analyse, Ouagadougou, Programme des Nations Unies pour le Développement.

LACHAUD J.P. (2000), « Dépenses des ménages, développement humain et pauvreté au Burkina-Faso : Substitution ou complémentarité? », Document de 
travail n49/2000, Centre d'Économie du Développement, Université Montesquieu Bordeaux IV.

LACHAUD J.P. (1999), «Envois de fonds, inégalité et pauvreté au Burkina Faso », Revue Tiers Monde, n²160, octobre-décembre.

LACHAUd J.P. (1997), Pauvreté, vulnérabilité et marché du travail au Burkina Faso, Série de recherche 2, Centre d'Économie du Développement, Université Montesquieu Bordeaux IV.

MAHIEU F.R., (1990), Les fondements de la crise économique en Afrique, Paris, l'Harmattan.

Ministère de l'Économie, des Finances et du Plan du Burkina Faso (2000), Poverty Reduction Strategy Paper, Ouagadoudou, mai, Burkina Faso.

Ministère de l'Économie, des Finances et du Plan du Burkina Faso et GTZ, (1995), Instrument Automatisé de Prévision. Manuel d'utilisation et note méthodologique, Ouagadoudou, mars, Burkina Faso.

Montaud J.M., (2002), «Ajustements structurels et inégalités des niveaux de vie en Afrique », dans Politiques sociales et croissance économique, Tome 1 , sous la direction de Jean-Marc DuPUIS et al, l'Harmattan.

Morrisson C., (1992), «Ajustement et Équité », Centre de développement de l'OCDE, Cahiers de Politique Économique, $\mathrm{n}^{\circ} 1$.

Moser C.O.N., (1996), "Confronting Crisis. A Comparative Study of Houshold Responses to Poverty and Vulnerability in Four Poor Urban Communities", Washington, ESD, $\mathrm{n}^{\circ} 8$.

PNUD, (1993, 2000), Rapport Mondial sur le Développement Humain, Bruxelles, De Boeck Université.

RAFFinot M., (1997), Stratégie nationale de réduction de la pauvreté : étude de cas du Burkina Faso, Université Paris IX-Dauphine.

RAVAllion M., (1998), "Poverty in Theory and Practice, Washington", LSMS, Working Papers $\mathrm{n}^{\circ} 103$, Banque Mondiale.

ROBILLIARD A.S (2000), «Trois outils pour l'étude d'une économie agricole pauvre : microéconométrie, modèle d'Équilibre Général Calculable et microsimulation appliqués à Madagascar », Thèse de Doctorat, Université Panthéon-Sorbonne.

SEN A. (1992), Inequality Rexamined, Oxford, Clarendon Press.

SEN A. (1985), Commodities and Capabilities, Amsterdam, North-Holland. 


\section{Annexe : liste des variables et des paramètres du modèle}

\begin{tabular}{|c|c|c|}
\hline Nom & Définition & Nombre \\
\hline$\overline{C D Q_{i, h}}$ & Volume de la consommation du ménage $h$ en produit domestique $i$ & 32 \\
\hline$C F D_{h}$ & Valeur de la consommation finale du ménage $h$ en biens domestiques & 8 \\
\hline$C F_{h}$ & Valeur de la consommation finale du ménage $h$ & 8 \\
\hline$C F M_{h}$ & Valeur de la consommation finale du ménage $h$ en biens importés & 8 \\
\hline$C M Q_{m, h}$ & Volume de la consommation du ménage $h$ en produit importé $m$ & 40 \\
\hline$D I Q_{i}$ & Volume de produit domestique $i$ destiné à la consommation intermédiaire & 4 \\
\hline$D S_{i}$ & Volume de l'offre de produit $i$ destinée au marché domestique & 4 \\
\hline$E_{i}$ & Volume des exportations de produit $i$ & 4 \\
\hline$I G D Q_{i}$ & Volume de l'investissement public en produit domestique $i$ & 4 \\
\hline$I G M Q_{m}$ & Volume de l'investissement public en produit importé $m$ & 5 \\
\hline$I P$ & Valeur de l'investissement privé & 1 \\
\hline$I P D Q_{i}$ & Volume de l'investissement privé en produit domestique $i$ & 4 \\
\hline$I P M Q_{m}$ & Volume de l'investissement privé en produit importé $m$ & 5 \\
\hline$L D_{i}$ & Volume de la demande de travail de la branche $i$ & 4 \\
\hline $\operatorname{Lnp} p_{i}^{D}$ & Volume de la demande de travail non protégé de la branche $i$ & 4 \\
\hline$M I Q_{m}$ & Volume de produit importé $\mathrm{m}$ destiné à la consommation intermédiaire & 5 \\
\hline$M_{m}$ & Volume des importations de produit $m$ & 5 \\
\hline$P D_{i}$ & Prix du produit domestique $i$ & 4 \\
\hline$P E_{i}$ & Prix des biens exportés par la branche $i$ & 4 \\
\hline$P M_{m}$ & Prix à la consommation des importations en produit $m$ & 5 \\
\hline$P_{n m}$ & Prix des services non marchands & 1 \\
\hline$P V A_{i}$ & Prix de la valeur ajoutée dans la branche $i$ & 4 \\
\hline$P V A_{n m}$ & Prix de la valeur ajoutée dans la branche non marchande & 1 \\
\hline$P X_{i}$ & Prix de la production dans la branche $i$ & 4 \\
\hline$R K a g$ & Revenu du capital agricole & 1 \\
\hline RKnag & Revenu du capital non agricole & 1 \\
\hline$S E$ & Valeur de l'épargne des entreprises & 1 \\
\hline$S G$ & Valeur de l'épargne des APU & 1 \\
\hline$S M_{h}$ & Valeur de l'épargne du ménage $h$ & 8 \\
\hline$w_{i}$ & Taux de salaire dans la branche $i$ & 4 \\
\hline$w_{n p}$ & Taux de salaire du travail non protégé & 1 \\
\hline$w_{p}$ & Taux de salaire du travail protégé & 1 \\
\hline$X_{i}$ & Volume de la production de la branche $i$ & 4 \\
\hline$X_{n m}$ & Volume de la production de services non marchands & 1 \\
\hline$Y E$ & Valeur du revenu total des entreprises & 1 \\
\hline$Y G$ & Valeur du revenu total des APU & 1 \\
\hline$Y M_{h}$ & Valeur du revenu total du ménage $h$ & 8 \\
\hline$L P$ & Ligne de pauvreté & 1 \\
\hline$E R$ & Taux de change (numéraire) & 1 \\
\hline
\end{tabular}


Variables exogènes

\begin{tabular}{llc}
\hline Nom & \multicolumn{1}{c}{ Définition } & Nombre \\
\hline$C N M_{G}$ & Valeur de la consommation finale de services non marchands des APU & 1 \\
$C N M_{h}$ & Valeur de la consommation de services non marchands du ménage $h$ & 8 \\
$I G$ & Valeur de l'investissement public & 1 \\
$K_{i}$ & Volume du stock de capital dans la branche $i$ & 5 \\
$L n p^{S}$ & Volume de l'offre totale de travail non protégé & 1 \\
$L p_{n m}$ & Volume de la demande de travail de la branche non marchande & 1 \\
$L p^{D} i$ & Volume de travail protégé de la branche $i$ & 4 \\
$P W E_{i}$ & Prix en devises des exportations en bien produit par la branche $i$ & 4 \\
$P W M_{i}$ & Prix en devises des importations en produit $i$ & 5 \\
$S F$ & Valeur de la capacité ou du besoin de financement du reste du monde & 1 \\
$T F M$ & Valeur en devise des transferts de fonds perçus par les ménages & 1 \\
$T G F$ & Valeur des transferts institutionnels perçus par le reste du monde & 1 \\
$T G M$ & Valeur des transferts institutionnels perçus par les ménages & 1 \\
$T E F$ & Transfert en capital des entreprises vers le reste du monde & 1 \\
$w_{p}$ & Taux de salaire du travail protégé & 1 \\
& &
\end{tabular}

Total variables exogènes 


\section{Paramètres}

$\sigma_{i}^{p} \quad$ Élasticité de substitution entre capital et travail dans la branche $i$

$\alpha_{i}^{p} \quad$ Paramètre factoriel de la fonction de production de la branche $i$

$A_{i}^{p} \quad$ Paramètre de dimension de la fonction de production de la branche $i$

$\sigma_{i}^{t} \quad$ Élasticité de transformation entre $E_{i}$ et $D_{i}$

$\alpha_{i}^{t} \quad$ Paramètre de la fonction CET de la branche $i$

$A_{i}^{t} \quad$ Paramètre de dimension de la fonction CET de la branche $i$

$\sigma_{f}^{l} \quad$ Élasticité de substitution entre travail qualifié et travail non qualifié dans la branche $i$

$\alpha_{i}^{l} \quad$ Paramètre de la fonction CES entre travail qualifié et travail non qualifié dans la branche $i$

$A_{i}^{l} \quad$ Paramètre de dimension de la fonction CES entre travail qualifié et travail non qualifié dans la branche $i$

$C_{\text {min }_{i, h}}$ Volume de la consommation incompressible en bien $i$ du ménage $h$

pmc $_{i, h} \quad$ Propension marginale à consommer le bien $i$ du ménage $h$

$l_{h, n q} \quad$ Dotation du ménage $h$ en facteur travail non qualifié

$l_{h, q} \quad$ Dotation du ménage $h$ en facteur travail qualifié du secteur privé

$l_{h, n m} \quad$ Dotation du ménage $h$ en facteur travail qualifié du secteur public

$t g_{h} \quad$ Part des transferts institutionnels redistribués au ménage $h$

$t f_{h} \quad$ Part des transferts de fonds perçus par le ménage $h$

$r_{h, a g} \quad$ Part des revenus du capital agricole destinés au ménage $h$

$r_{h, n a g}$ Part des revenus du capital non agricole destinés au ménage $h$

re Part des revenus du capital destinés aux entreprises

$r e_{n a g} \quad$ Part des revenus du capital non agricole destinés aux entreprises

$t m_{h}$ Taux d'imposition directe sur le revenu du ménage $h$

tye Taux d'imposition directe sur le revenu des entreprises

$t x_{i} \quad$ Taux d'imposition sur la production de la branche $i$

$t m_{i} \quad$ Taux d'imposition sur les importations de produits $i$

$s m_{h} \quad$ Propension moyenne à épargner du ménage $h$

$a m_{m j} \quad$ Coefficients techniques de Leontiev en bien importé $m$ pour la branche $j$

$a d_{i j} \quad$ Coefficients techniques de Leontiev en bien domestique $i$ pour la branche $j$

$\iota g d_{i} \quad$ Clés de répartition de l'investissement public entre les biens domestiques $i$

$\iota p d_{i} \quad$ Clés de répartition de l'investissement privé entre les biens domestiques $i$

$\iota g m_{m} \quad$ Clés de répartition de l'investissement public entre les biens importés $m$

$\iota p m_{m} \quad$ Clés de répartition de l'investissement privé entre les biens importés $m$

$\emptyset_{m, h} \quad$ Clés de répartition de la consommation finale des ménages en biens importés $m$

$\beta_{i} \quad$ Poids du bien domestique $i$ dans la ligne de pauvreté

$\beta_{m} \quad$ Poids du bien importé $m$ dans la ligne de pauvreté 